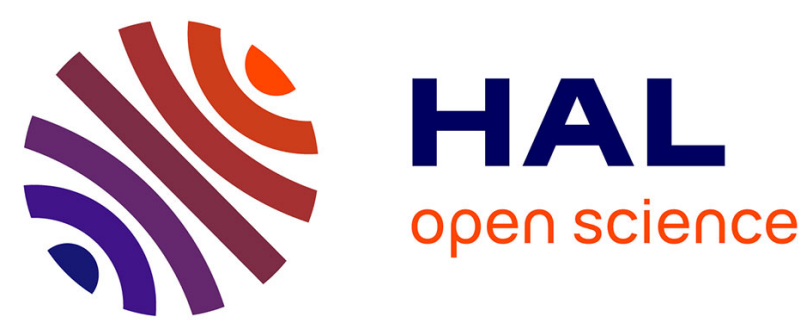

\title{
Developments and Control of Biocompatible Conducting Polymer for Intracorporeal Continuum Robots
}

\author{
Mohamed Taha Chikhaoui, Amine Benouhiba, Patrick Rougeot, Kanty \\ Rabenorosoa, Morvan Ouisse, Nicolas Andreff
}

\section{- To cite this version:}

Mohamed Taha Chikhaoui, Amine Benouhiba, Patrick Rougeot, Kanty Rabenorosoa, Morvan Ouisse, et al.. Developments and Control of Biocompatible Conducting Polymer for Intracorporeal Continuum

Robots. Annals of Biomedical Engineering, 2018, 1, pp.1 - 11. 10.1007/s10439-018-2038-2 . hal02130056

\section{HAL Id: hal-02130056 \\ https://hal.science/hal-02130056}

Submitted on 15 May 2019

HAL is a multi-disciplinary open access archive for the deposit and dissemination of scientific research documents, whether they are published or not. The documents may come from teaching and research institutions in France or abroad, or from public or private research centers.
L'archive ouverte pluridisciplinaire HAL, est destinée au dépôt et à la diffusion de documents scientifiques de niveau recherche, publiés ou non, émanant des établissements d'enseignement et de recherche français ou étrangers, des laboratoires publics ou privés. 


\title{
Developments and Control of Biocompatible Conducting Polymer for Intracorporeal Continuum Robots
}

\author{
Mohamed Taha Chikhaoui*2, Amine Benouhiba*1, Patrick \\ Rougeot $^{1}$, Kanty Rabenorosoa ${ }^{1}$, Morvan Ouisse ${ }^{1}$, and Nicolas \\ Andreff $^{1}$ \\ ${ }^{1}$ FEMTO-ST Institute, Univ. Bourgogne Franche-Comté/CNRS/ENSMM, \\ Besançon, France \\ ${ }^{2}$ Laboratory for Continuum Robotics, Leibniz Universität Hannover, \\ Hanover, Germany \\ chikhaoui@lkr.uni-hannover.de \\ * These authors equally contributed to the manuscript.
}




\begin{abstract}
Dexterity of robots is highly required when it comes to integration for medical applications. Major efforts have been conducted to increase the dexterity at the distal parts of medical robots. This paper reports on developments towards integrating biocompatible conducting polymers into inherently dexterous concentric tube robot paradigm. In the form of trilayer thin structures, these micro-actuators produce high strains while requiring less than 1 volt for actuation. Fabrication, characterization, and first integrations of such micro-actuators are presented. The integration is validated in a preliminary telescopic soft robot prototype with accurate position control with trajectory tracking scenarios. Further, the micro-actuator is controlled for laser steering with displacements up to $5 \mathrm{~mm}$. Our first developments aim towards intracorporeal medical robotics, with miniaturized actuators to be embedded into continuum robots.
\end{abstract}

Keywords: conducting polymers, micro-actuators, continuum robots, medical robotics, position control.

\title{
1. INTRODUCTION
}

During the last ten years, the development of continuum robots for minimal invasive surgery (MIS) and natural orifice transluminal endoscopic surgery (NOTES) has been increasing and numerous innovative designs have been introduced. ${ }^{1}$ Despite of the breakthrough of continuum robots, the actuation of an endomicroscope tip is a major technological challenge. ${ }^{10}$ In the realm of gastro-intestinal endoscopy, this possibility brings high potential for accurate navigation and diagnosis as closely as possible to the tissue. Among the different candidate designs for such intracorporeal applications, concentric tube robots (CTR) present one of the highest abilities. In contrast with tendon-driven robots ${ }^{19,17}$ or pneumatically actuated robots, ${ }^{6}$ CTR are distinguished by their miniaturization (outer diameters smaller than $3 \mathrm{~mm}$ ), dexterity (their degrees of freedom increase by adding more component tubes), reachability (extension and contraction possibilities), as well as function integration with their free lumen. ${ }^{16,11}$ Nevertheless, CTR motion and dexterity can be limited by their preset component tubes' curvatures. While the overall curvature of a given segment of a CTR can be modified online by concentrically arranging and rotation individual tubes [AnorICRA2011], it has been shown (i) that for the same number of tubes, varying the curvature along one or two directions increases both the workspace and the robot's manipulability ${ }^{3}$, and (ii) that combining concentric tubes with tendon-actuated segments highly improves motion abilities such as follow-the-leader deployment [Amanov ICRA 2017] and dexterity [WuRAL2017,LiMechMT2017].

Anor T, Madsen J, Dupont P. Algorithms for design of continuum robots using the concentric tubes approach: A neurosurgical example. IEEE International Conference on Robotics and Automation, 2011, 667-673.

Amanov E, Granna J, Burgner-Kahrs J. Toward Improving Path Following Motion: Hybrid Continuum Robot Design. IEEE International Conference on Robotics and Automation, 2017, 4666-4672.

Wu L, Crawford R, Roberts J. Dexterity Analysis of Three 6-DOF Continuum Robots Combining Concentric Tube Mechanisms and Cable-Driven Mechanisms. IEEE Robotics and Automation Letters 2017, 2(2), 514-521. 
$\mathrm{Li}$ Z, Wu L, Ren H, Yu H. Kinematic comparison of surgical tendon-driven manipulators and concentric tube manipulator. Mechanism and Machine Theory 2017, 107, 148165.

In order to reduce invasiveness of medical/surgical operations and to improve standard endoscopes' accuracy, this paper introduces a novel biocompatible microactuation technique of tubes able to house diagnosis tools to perform fiber-based optical biopsy or laser surgery. The possible embedded techniques of micro-actuation include hydraulic actuation, ${ }^{12}$ shape memory alloys, ${ }^{8}$ and piezoelectric actuators, ${ }^{13}$ among others. The major drawbacks of these techniques for our target application comprise the complexity of the control, the additional heat, or the need for high power. ElectroActive Polymers (EAP), and particularly Conducting Polymers (CP) were selected for several reasons. Indeed, they require very low operating voltages $(<2 \mathrm{~V})$ while providing large deformations, biocompatibility, softness, and high miniaturization potential. ${ }^{2}$ These actuators can undergo volume change by applying a potential of reduction or oxidation onto the component electrodes, which results in the expansion or contraction of the polymer by absorption or expulsion of ions. The latter are provided by a surrounding electrolyte in aqueous form or as gel to allow for encapsulation. One of the less energy consuming CP, PolyPyrrole (PPy) is studied hereby. The challenge is to bend tubes with diameters smaller than $1.5 \mathrm{~mm}$ with PPy-based micro-actuators of approximately $10 \mu \mathrm{m}$ in thickness for use in active endomicroscopy. The kinematic benefits of such an actuation were studied in combination with concentric tube paradigm $^{3}$ and the first integration of such actuators in a soft robot was introduced. ${ }^{4}$ The general concept is depicted in Figure 1.

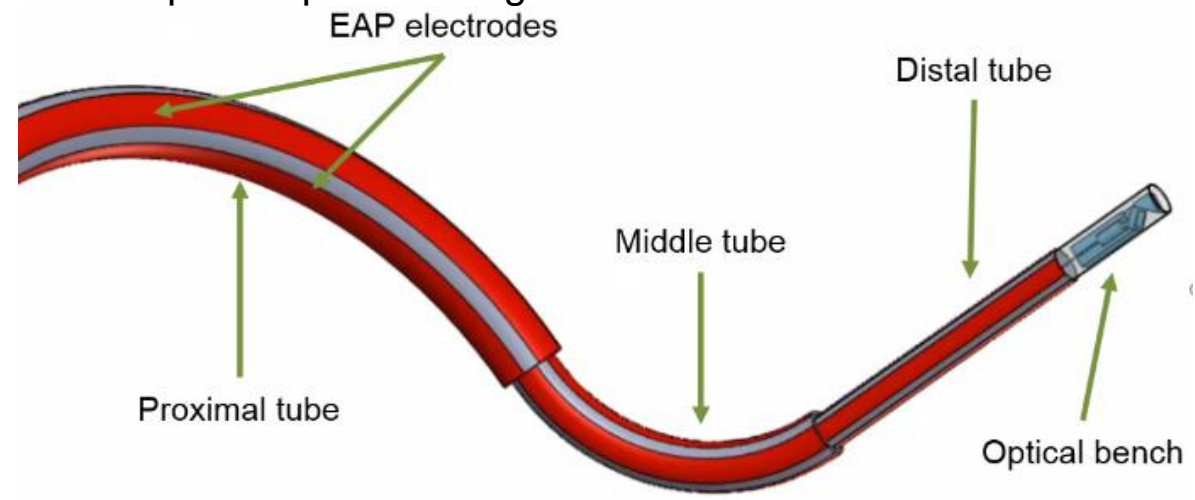

Figure 1: Concept of a continuum robot founded on concentric tube paradigm with variable curvatures using soft micro-actuators based on EAP. An optical bench is embedded in the robot's end-effector as an example. ${ }^{5}$

Added to summarizing methods of fabrication of EAP-based micro-actuators, this paper introduces two integration scenarios of such actuators. Extended analysis and experiments with a 2D soft robot prototype are provided. We further extend our previous investigations, ${ }^{5}$ to include laser steering and its accurate control. In the following, the technological developments are detailed and validated through experimental results.

\section{MATERIALS AND METHODS}

Trilayer micro-actuators: fabrication and actuation principle 
All micro-actuator samples are constructed by electrochemical polymerization process using a Voltastat PGP 201 potentiostat (Radiometer Analytical, Loveland CO, USA) controlled by VoltaMaster 4 software. A standard three-electrode system is linked to the potentiostat and composed by $\mathrm{Ag} / \mathrm{AgCl}$ electrode as reference electrode and a platinum sheet as counter-electrode. The working electrode is a PolyVinylidene DiFluoride (PVDF) membrane Immobilon-P (Merck Millipore Corp., Darmstadt, Germany) of $110 \mu \mathrm{m}$ in thickness. According to product specifications, the membrane is hydrophobic and presents pore size of $0.45 \mu \mathrm{m}$ with a porosity rate $70 \%$. The interest in using this membrane relies on the pores, thus it can absorb and permits transport of electrolyte during actuation. Prior to its introduction in the polymerization setup, conductance of the membrane is supplied by vacuum plasma sputtering of $40 \mathrm{~nm}$ chromium/gold layer on both sides. Further, the electrochemical deposit is performed based on Yamaura et al. protocol ${ }^{18}$ and Gairhe et al. process. ${ }^{9}$ Thus, low oxidation potential $(<1 \mathrm{~V})$ and current density $\left(0.1 \mathrm{~mA} / \mathrm{cm}^{2}\right)$ are applied, while operating at low temperature $\left(\sim-25^{\circ} \mathrm{C}\right)$. In order to provide better adherence and compactness to the polymer layer, organic solvent is selected, namely Bis(TriFluoromethane) Sulfonlmide Lithium salt (LiTFSI) at $0.05 \mathrm{M}$ and $99 \%$ pure pyrrole at $0.06 \mathrm{M}$ (Sigma Aldrich, Saint Louis, USA). The fabrication process is summarized in Figure 2a and concerns the deposition of conductive electrodes by plasma sputtering in clean room conditions, prior to electrochemical deposition of PPy layers.

a)

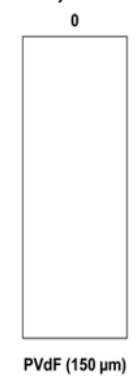

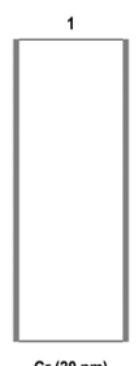

$\operatorname{Cr}(20 \mathrm{~nm})$

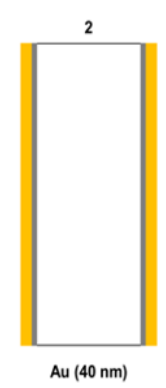

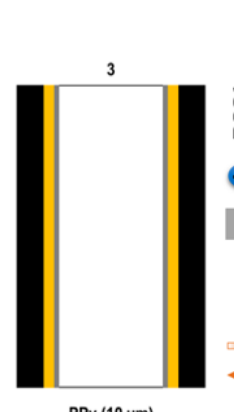

PPy $(10 \mu \mathrm{m})$ b)

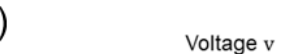

Voltage

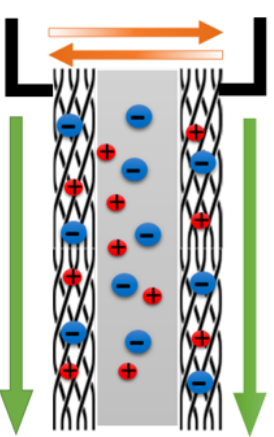

Figure 2: a) Fabrication flowchart of the tri-layer PPy-based micro-actuator and b) their actuation principle.

The actuation principle of PPy-based micro-actuators is depicted in Figure 2b. The electrical stimulation through the voltage $v$ produces a chemical reaction (oxidation and reduction). The ions of the electrolyte then migrate on one electrode or the other, depending on the polarity of the voltage. In such electrolytes used for EAP, the anions are larger than cations. Due to ion migration, a difference in volume is produced, which creates the bending motion of the actuator.

After synthesis, a micro-actuator of $40 \times 1 \times 0.16 \mathrm{~mm}^{3}$ is patterned. For actuation, the sample is immersed in the actuating solution composed by $0.05 \mathrm{M}$ of LiTFSI diluted in propylene carbonate.

It is worth mentioning that the porosity of the PVDF membrane is a key feature. Indeed, not only the membrane is (i) a passive support holding both conductive electrodes, it also acts as (ii) a container for the actuation electrolyte, which then enables storing and transporting the ions required for the reversible chemical reaction.

\section{Position control of a 2D soft robot}


A 2D telescopic soft robot is designed and presented in Figure 3a. It is constituted of a straight section (stiff tube) of $3.8 \mathrm{~mm}$ in length, and a curved section (tri-layer planar $\mathrm{PVDF} / \mathrm{Cr} / \mathrm{Au} / \mathrm{PPy}$ micro-actuator). The latter can translate in the tube along Y-axis and bend around Z-axis. The forward in-plane kinematic model (FKM) is based on the constant curvature assumption, as will be shown for the developed micro-actuator. An arc of a circle in the $X Y$ plane is defined, following, by its curvature $\mathrm{k}$ (inverse of the radius of curvature $r$ ) and its length $\ell$.

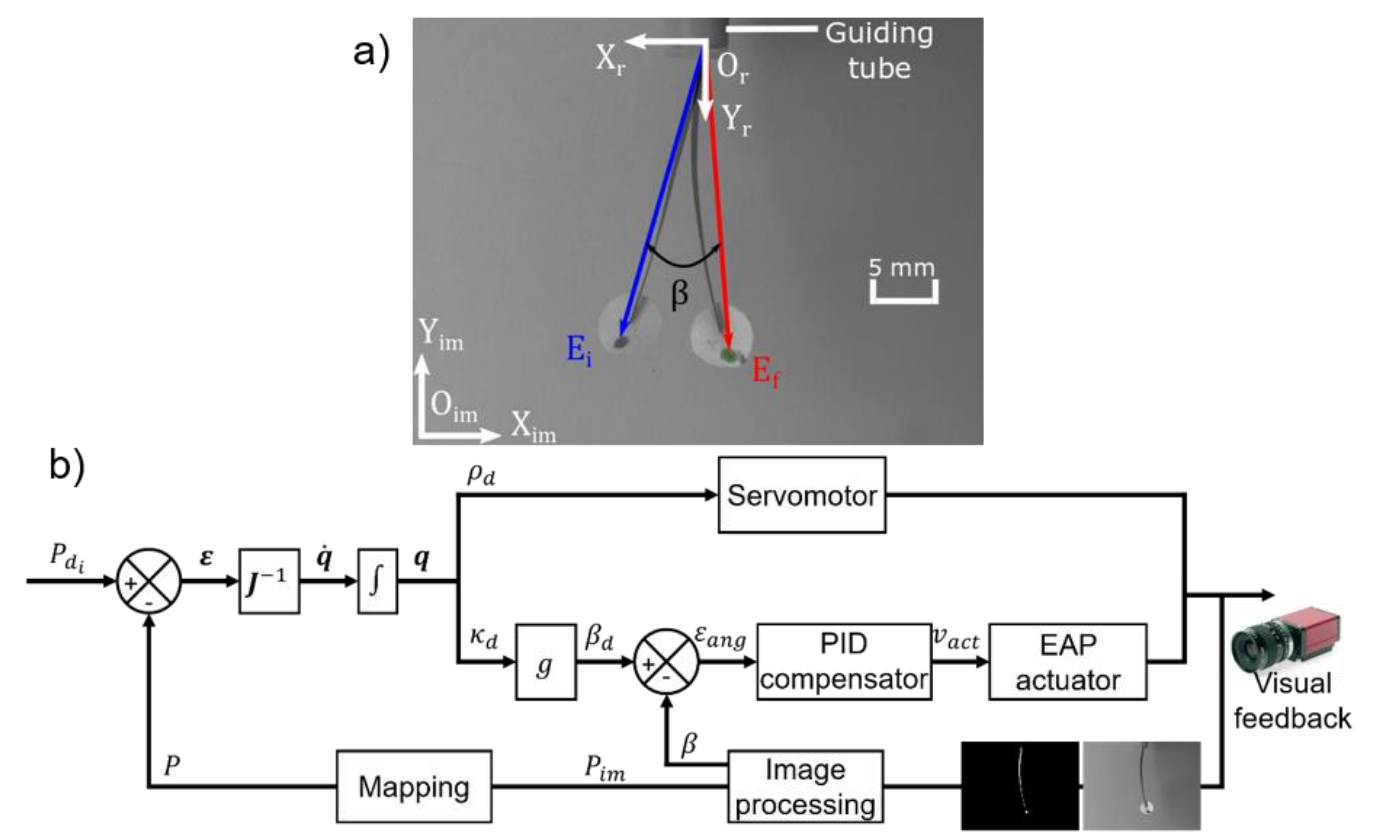

Figure 3: a) Telescopic soft robot design and b) its position control diagram. ${ }^{4}$

Thus, the transformation matrix from the arc origin frame $O$ to its tip frame $E$ is:

$$
{ }^{0} \boldsymbol{T}_{1}=\left[\begin{array}{cc}
\boldsymbol{R}_{z}(\theta) & \boldsymbol{p} \\
\mathbf{0} & 1
\end{array}\right]
$$

where $\boldsymbol{R}_{Z}(\theta)$ is the rotation about the Z-axis with the bending angle $\theta=\kappa \ell$ and $\boldsymbol{p}=$ $\left[\begin{array}{lll}r(1-\cos \theta) & r \sin \theta\end{array}\right]^{T}$ is the arc tip location. The arc variables $\mathrm{K}$ and $\ell$ are linked to the robot actuators $\boldsymbol{q}=\left[\begin{array}{ll}\rho & v\end{array}\right]^{T} . \rho$ is the translation actuator and $v$ is the actuation voltage of the micro-actuator. The intrinsic curvature of the second segment is denoted $\kappa_{2 i n}=$ $C_{P P y} v$, with $C_{P P y}$ the PPy electro-chemical conversion of the micro-actuator. The microactuator's stiffness is considered negligible compared to that of the tube. The length $\ell_{1}=L_{1}$ of the first segment (guiding tube) is constant. The second segment's curvature is $\kappa_{2}=\kappa_{2 i n}$ as it is free outside the guiding tube and its length is $\ell_{2}=L_{2}$.

The control diagram is detailed in Figure 3b. The reference trajectory is discretized into successive desired point $\left(P_{d_{i}}\right)$ and this reference is then compared to the measured position through camera feedback and image processing. A differential inverse kinematic based controller is then utilized to compensate for the error and update the actuators. The translation actuation is controlled through the motor's inner loop. Concerning the curvature, a PID controller is applied after a transformation to angular displacement. The experimental setup includes the voltage controller, the translation stage, and the camera feedback. The latter components are monitored through a Matlab Simulink program using the Visual Servoing Platform (ViSP: https://visp.inria.fr/) through the new opensource dedicated blockset CVLink 
(https://sourcesup.renater.fr/cvlink/). Matlab/Simulink $2011 \mathrm{~b}$ is ran on an Intel Xeon CPU 2.33 GHz computer. Tracking the position of the actuator is performed using an IEEE 1394 Guppy FireWire camera (https://www.alliedvision.com/en/digital-industrialcamerasolutions.html) placed in front of the smallest dimension of the actuator (hence its thickness) as shown in Figure 3a. The actuator is attached from both sides via a miniaturized conductive gripper. The latter is fabricated from a conductive tube of $1.5 \mathrm{~mm}$ diameter and $30 \mathrm{~mm}$ length. The tube is then severed longitudinally through electrical discharge machining (wire erosion) using a fine tool-electrode of $100 \mu \mathrm{m}$. Therefore, two separate arms are obtained. A heat-shrinkable sleeve of $1 \mathrm{~mm}$ length is wrapped around the two gripper's arms at their middle to unite them and the actuator is properly clamped at one tip. Thus, the required displacement is obtained and the micro-actuator is properly connected. Both of the gripper arms are finally welded to the power wiring. The potential is supplied using the USB-6211 National Instruments multifunction data acquisition module (www.ni.com/). The two analog outputs used to send the voltages can operate between $+/-10 \mathrm{~V}$ with a resolution of $3.512 \mathrm{mV}$. One analog input is used to measure the current with a quantum of $0.088 \mathrm{mV}$ through a 10 $\Omega$ resistor. On the other side, the gripper is linked to the M111.1DG translation stage from Physik Instrumente (Karlsruhe, Germany) with a travel range of $25 \mathrm{~mm}$, a maximum velocity of $1.5 \mathrm{~mm} / \mathrm{s}$, and a minimum incremental motion of $0.05 \mu \mathrm{m}$ using the Mercury C-863 single-axis controller (http://www.physikinstrumente.com/). Therefore, the miniaturized conductive gripper and the actuator can translate inside a guiding tube of $5 \mathrm{~mm}$ in diameter and $20 \mathrm{~mm}$ in length.

Using the visual feedback, the program detects the actuator by means of a suitable thresholding. In order to track exclusively the actuator end-effector, a physical marker is joined as demonstrated in Figure 3a. It is a circular marker with a dark point of approximately $1 \mathrm{~mm}$ diameter $(2.5 \%$ of the actuator's length) that can be properly detected and tracked by the camera. A preliminary test on the detected mark area is done and an additional evaluation of its shape factor is performed through the isoperimetric quotient (circularity). This test permits a robust tracking of the endeffector during the experiments even when the scene is subject to luminosity variation. The measurement accuracy depends substantially on the marker size. In order to quantify the former, the actuator is placed at a static position, and the marker center is detected for an arbitrary duration. Following the calibration of the camera, the variation of the position is detected and converted afterwards to millimeters. The horizontal error of the marker barycenter is about $33 \mu \mathrm{m}$ while the vertical error is $22 \mu \mathrm{m}$. In order to enable a suitable description of the micro-actuator's position with a single variable, we further defined its angular position $\beta$ (see Figure 3a). This variable is used in the control algorithm as well as in the following characterizations.

\section{Laser steering using trilayer micro-actuators}

In this subsection, we are interested in the closed-loop control of an optical fiber (SMF 28) by PPy micro-actuation. The micro-actuator is fixed to the optical fiber by small pieces of heat-shrinkable sleeve (one at each end of the micro-actuator). By doing so, decent deflection angles of the system were obtained, as shown in Figure $4 a$. 


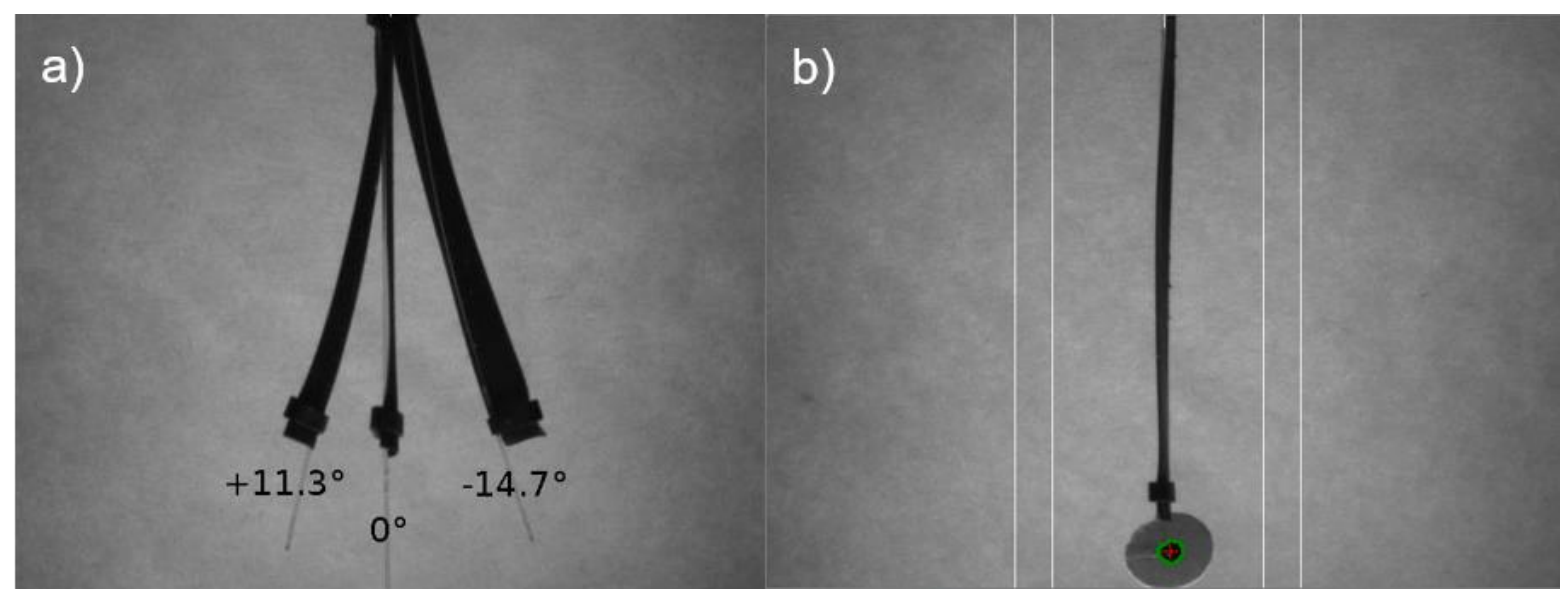

Figure 4: a) Example bending angles of the optical fiber by the PPy micro-actuator, with an actuation voltage of $-/+2 \mathrm{~V}$ from left to right and $b$ ) position tracking target placed on the free end of the optical fiber, used for visual tracking of the system.

As mentioned above, a Matlab Simulink program through a Visual Servoing Platform is used to process the camera feedback. The latter was used for the position tracking of a target placed on the free end of the optical fiber (see Figure $4 \mathrm{~b}$ ). The same power supply mechanism used in the previous section is applied here.

\section{RESULTS}

\section{Motion and force characterization of the micro-actuator}

The displacement characterization of the actuator was performed. First, the objective is to obtain the time response of the tri-layer actuator for different applied voltages (step response) in terms of angular displacement of its end-effector. The setup, previously described, is used to measure the deformation of the whole actuator. The offline image processing enables the angle detection according to the time as shown in Figure 5a. It is observed that the angle depends on the applied voltage and the response time is difficult to measure due to the drift. The system is nonlinear and the response time is variable as depicted in Figure $5 \mathrm{~b}$.
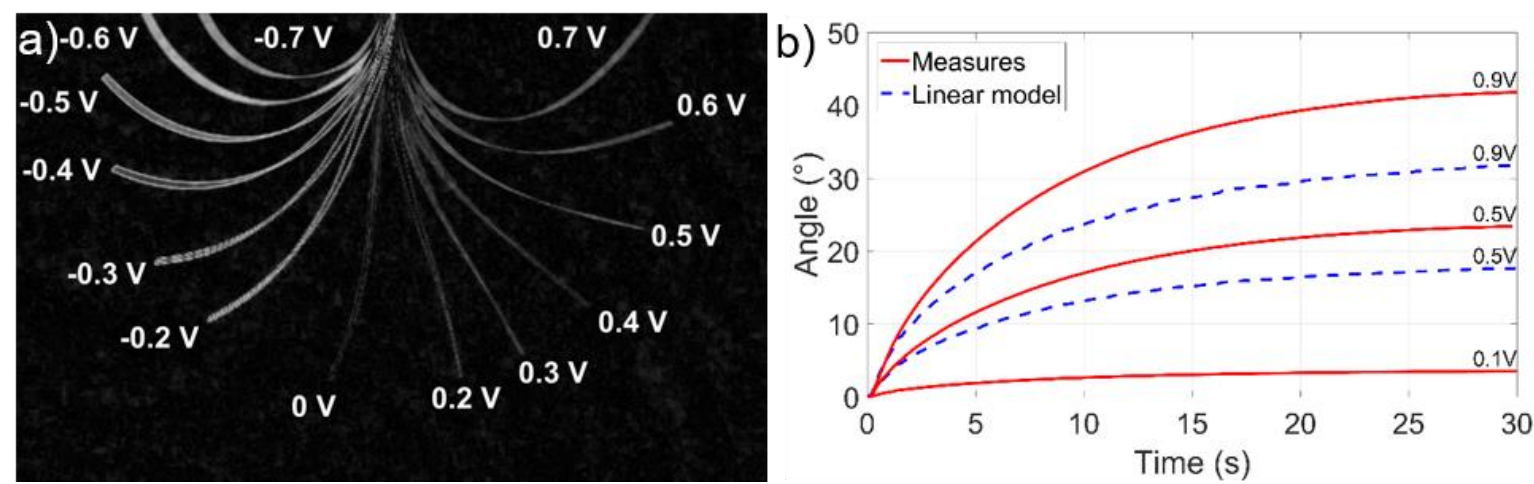

Figure 5: a) Actuation results through superimposition of different states. ${ }^{7}$ b) Open loop response with comparisons to a linear model.

Force measurements are performed using the experimental setup depicted in Figure 6 a. The micro-actuator is fixed to a conductive gripper ensuring both mechanical fixation and power supply. A FT-S-1000 force sensor (FemtoTools AG, Buchs, Switzerland), shown in Figure 6b, provides up to $1000 \mathrm{mN}$ measurement range with a resolution of $0.05 \mu \mathrm{N}$ at $10 \mathrm{~Hz}$. Further, a camera is set in order to monitor the 
experiments and to align the actuator movement with the sensor's probe sensitive direction (see Figure 6a). The latter works in compression and traction and has a width of $50 \mu \mathrm{m}$, consistent with the actuator's width of $2 \mathrm{~mm}$ to ensure full contact during motion. Control power and data acquisition are performed via a Matlab/Simulink program. Measurements are ensured to start at the closest possible position to the probe to prevent disturbances due to dynamics effects.

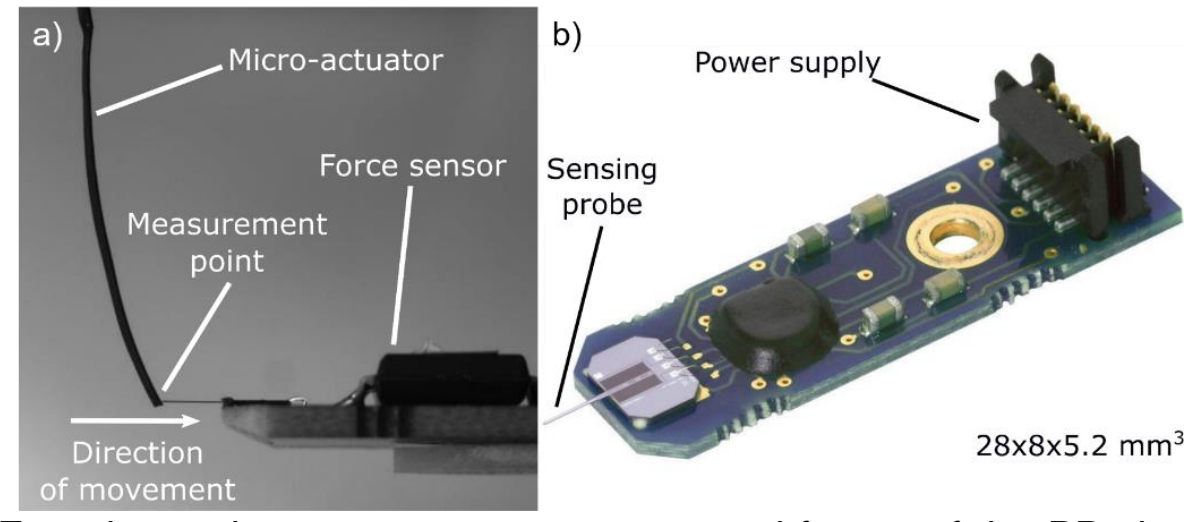

Figure 6: Experimental setup to measure generated forces of the PPy based microactuator. a) Actuator at a measurement configuration and b) force sensor. c) Impact of polymerization time on maximum generated forces at the micro-actuator tip.

Over incremental actuation voltages ranging from 0.2 to $2.2 \mathrm{~V}$, the generated forces were measured at the micro-actuator's tip. In order to avoid dynamic effects, a step input should be avoided. Instead, an incremental actuation of 55 seconds was chosen before reaching the reference voltage, as shown in Figure 7a. We believe this duration to be sufficiently high to stabilize the micro-actuator's motion. Therefore, for each polymerization time (2, 6, and 14 hours), three sets of measurements were performed and their mean value is considered. Figure 7 a depicts the example of a polymerization time of 10 hours. The results show that the generated force is proportional to the actuation voltage for a given polymerization time, but saturate at high voltages. This can be explained by the saturation in the ions' motion involved in the bending movement of the micro-actuators (cf. Figure 2b). Based on force measurements, we extracted the maximum forces for every polymerization time, as shown in Figure 7b. Maximum force measures range from 175 to $425 \mu \mathrm{N}$ and are proportional to the polymerization time as well. 

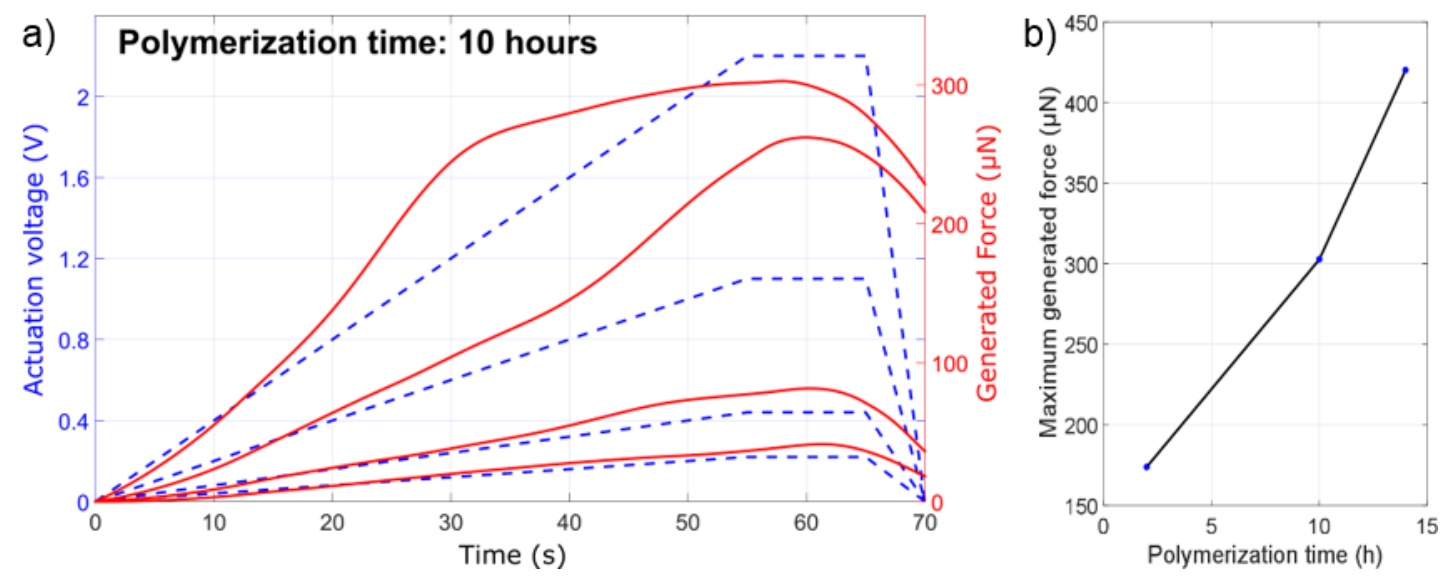

Figure 7: Study of polymerization time effect: a) Evolution of the generated forces (solid lines) for different actuation voltages (dashed lines) for a polymerization time of 10 hours, and b) maximum generated forces at the micro-actuator tip.

\section{Closed-loop control performances of the 2D soft robot}

Closed-loop control experiments were led using the 2D soft robot prototype such that $L_{1}=3.8 \mathrm{~mm}$. First, the robot's workspace is experimentally characterized, as depicted in Figure $8 \mathrm{a}$ with the inner and outer boundaries. The translation stage is driven between $\rho_{\min }=0 \mathrm{~mm}$ and $\rho_{\max }=25 \mathrm{~mm}$, while voltages ranging between $v_{\min }=$ $-0.7 \mathrm{~V}$ and $v_{\max }=0.7 \mathrm{~V}$ are applied to the micro-actuator. This characterization permitted to identify the workspace of interest in the following validation.

The control scheme previously presented in Figure $3 \mathrm{~b}$ was implemented to the considered robot using a sampling time of $0.2 \mathrm{~s}$. This is the minimum admissible by the Matlab/Simulink program with the running processor. The studied trajectories are located in the central lower part of the robot's workspace and concern a square path and a spiral. These trajectories consist of multiples intermediate desired points $P_{d_{i}}$ with timestamps. Each test is repeated three times to validate the repeatability of the process. The performances of trajectory tracking are quantified in terms of root mean square error (RMS), standard deviation (StD), and maximum error (Max). Table 1 summarizes these results, while Figure $8 \mathrm{~b}$ and Figure $8 \mathrm{c}$ depict and example of each trajectory performed by the $2 \mathrm{D}$ soft robot.

Table 1: Trajectory tracking errors with the 2D soft robot.

\begin{tabular}{|l|l|l|l|l|l|}
\hline \multirow{2}{*}{ Trajectory } & $\begin{array}{l}\text { Test } \\
\text { number }\end{array}$ & RMS $(\mu \mathrm{m})$ & StD $(\mu \mathrm{m})$ & $\operatorname{Max}(\mu \mathrm{m})$ & Time $(\mathrm{s})$ \\
\hline \multirow{3}{*}{ Square } & 1 & 221 & 201 & 458 & 420 \\
\cline { 2 - 6 } & 2 & 223 & 201 & 700 & 420 \\
\cline { 2 - 6 } & 3 & 273 & 180 & 597 & 420 \\
\hline \multirow{3}{*}{ Spiral } & 1 & 92 & 70 & 370 & 1000 \\
\cline { 2 - 6 } & 2 & 93 & 80 & 318 & 1000 \\
\cline { 2 - 6 } & 3 & 90 & 86 & 227 & 1000 \\
\hline
\end{tabular}



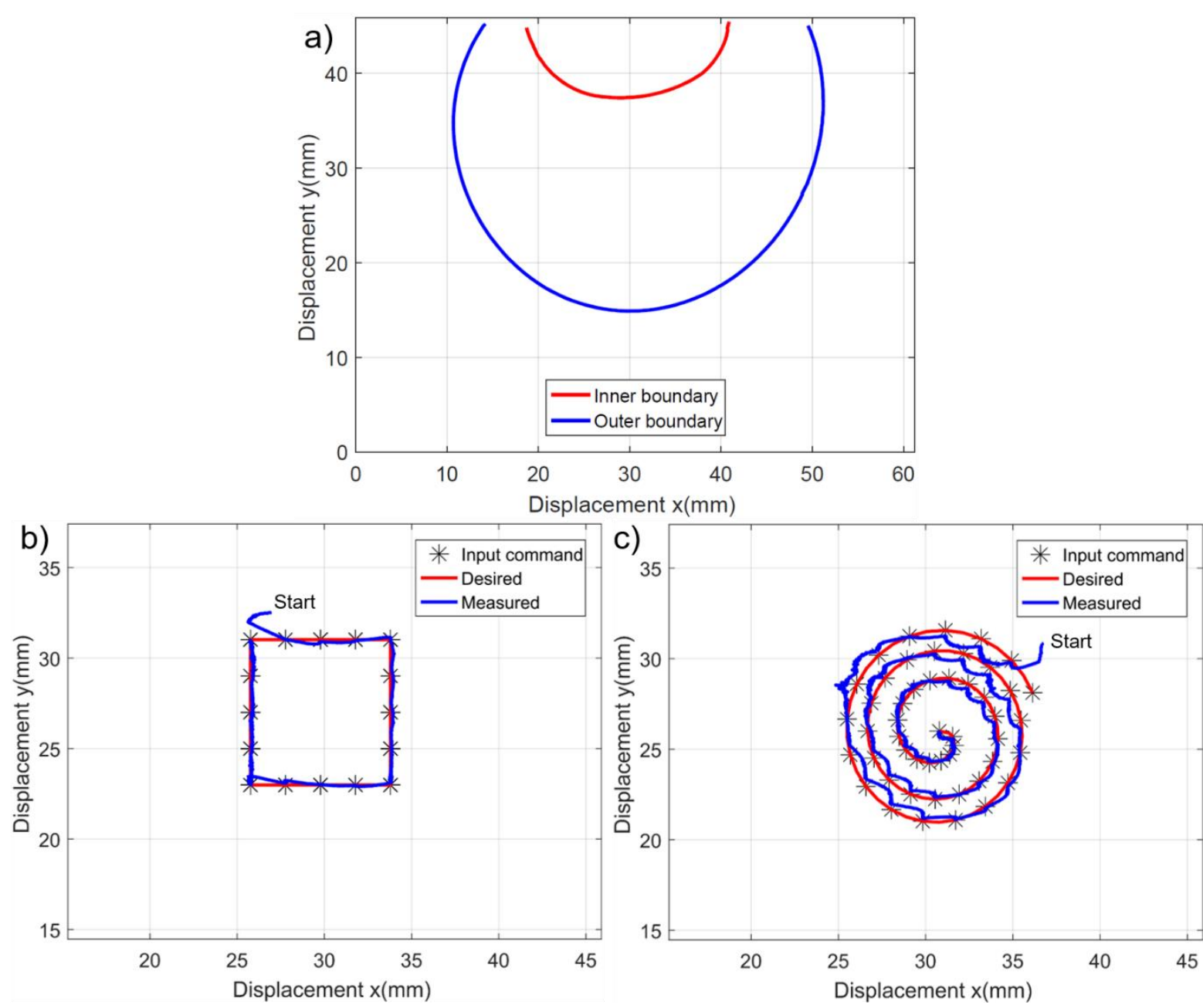

Figure 8: a) Experimental characterization of the workspace boundaries of the 2D soft robot, and trajectory tracking for b) square and c) spiral trajectories.

These results highlight a positioning accuracy with a mean RMS error of $239 \mu \mathrm{m}$, a mean StD of $194 \mu \mathrm{m}$, and maximum error of $700 \mu \mathrm{m}$ over the three tests of the square trajectory tracking. The overall mean RMS error, mean StD, and maximum error of the spiral trajectory tracking are $91.7 \mu \mathrm{m}, 78.6 \mu \mathrm{m}$, and $370 \mu \mathrm{m}$, respectively. The latter positioning performances are more accurate - more than 2.5 times - than the former as the sampling of the square trajectory is finer (i.e. the distance between two consecutive desired points $P_{d_{i}}$ is higher). Furthermore, the non-smoothness of the former trajectory (square) can explain the lower accuracy as the desired motion direction suddenly changes at the square's edges. However, the spiral trajectory tracking was around 2.38 times slower than the square trajectory tracking. One can conclude from these results that the trajectory tracking with the $2 \mathrm{D}$ soft robot can be achieved at different rates and different accuracy. The suitable feature for the intended application needs to be underlined based on safety requirements as the environment constraints (e.g. minimized error to avoid undesirable contact with the tissue) or the target application (e.g. fast motion for laser scanning).

\section{PPy actuation for laser steering}

The response of the PPy micro-actuator linked to the fiber in respect to the control references (step and stairs signals) is displayed in Figure 9a, Figure 9b, and Figure 
9c. The control voltage was limited to $+/-2.5 \mathrm{~V}$ in order to avoid any damage to the PPy micro-actuator due to high voltages.

Table 2 Quantitative evaluation of the angular responses for different input signals.

\begin{tabular}{|l|c|c|c|}
\hline \multirow{2}{*}{ Input signal } & \multirow{2}{*}{ Amplitude $\left(^{\circ}\right)$} & \multicolumn{2}{|c|}{ Position control error } \\
\cline { 3 - 4 } & & Mean (\%) & St. D (\%) \\
\hline Stairs & 0 to 5 & 1.5 & 0.38 \\
\hline Sawtooth & $+/-5$ & 8 & 0.55 \\
\hline
\end{tabular}

In the case of the step control signals, the system response is slower than that of the individual micro-actuator. This is an expected effect as bending the optical fiber requires more force with respect to the voltage limits. However, once the steady state is reached, the system fulfills the control requirements with a steady state error of less than $0.6 \%$. Moreover, we can see from Figure 9 that the system reaches the steady state slightly faster, in the case of the negative reference signal compared to the positive reference. This is due to the fact that fabrication of a tri-layer PPy microactuator with a perfectly symmetrical structure is still a major technological challenge. As for the stair reference signal (shown in Figure 9b), we can observe a similar behavior (the step reference) in terms of delay to reach the steady state. Nonetheless, in this case it is physically more constraining to bend from $2.5^{\circ}$ to $5^{\circ}$ than from $0^{\circ}$ to $2.5^{\circ}$. This behavior can be motivated by the fact that it is easier to produce bending near the relaxed state (at $0^{\circ}$ ) than after constraining the PPy micro-actuator with additional stiffness of the optical fiber.

a)

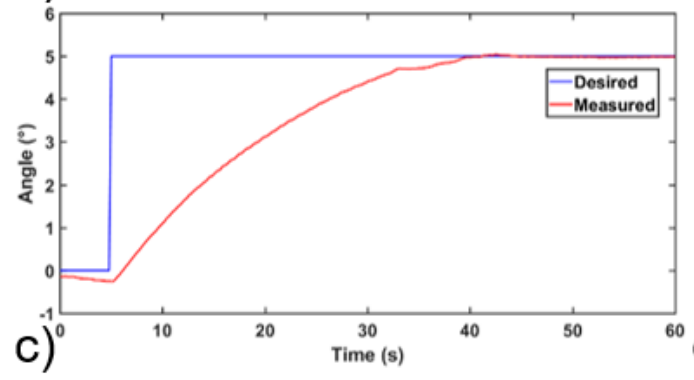

b)

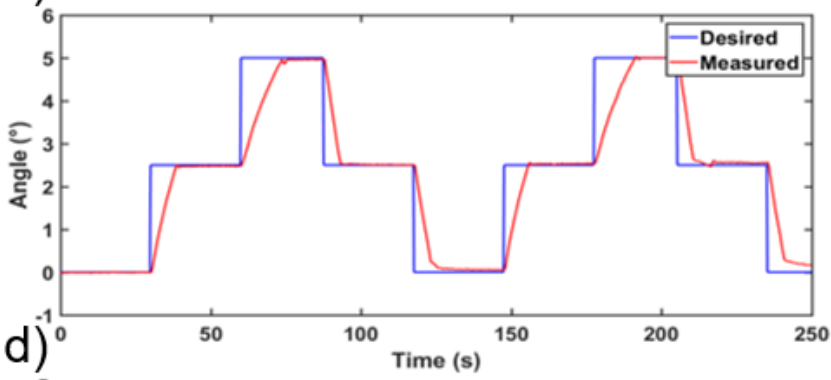

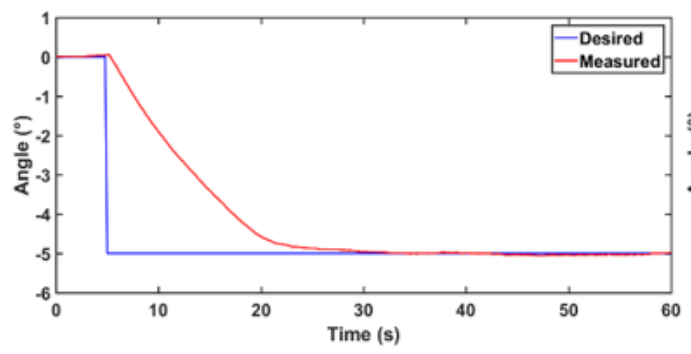

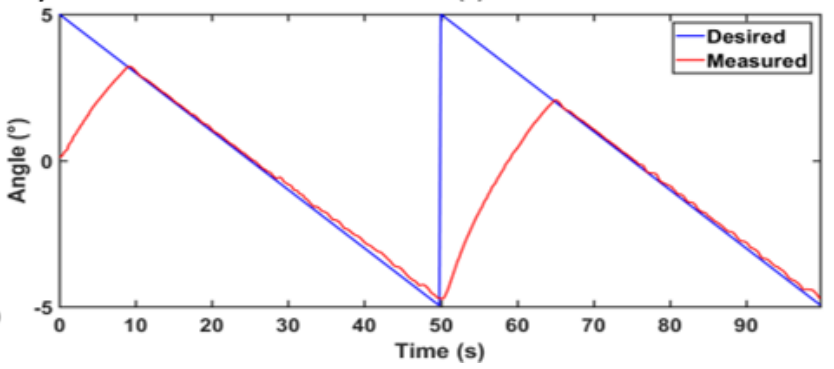

Figure 9: Angular response of the system to different reference signals, namely a) positive step, c) stairs, b) negative step, and d) sawtooth.

Further angular control experiments concerned the response of the system to sawtooth reference signal, with the same input voltage limit of $+/-2.5 \mathrm{~V}$. Figure $9 \mathrm{~d}$ shows that sudden reference switches (from $0^{\circ}$ to $5^{\circ}$ and from $-5^{\circ}$ to $5^{\circ}$ ) significantly decrease the tracking performances. That is why we can observe a dramatic jump in the mean error from $1.5 \%$ to $8 \%$ compared to the stairs reference signal (as shown 
in Table 2). When the reference signal is smoother, the system's tracking performances increase.

\section{DISCUSSION}

The large displacements of PPy-based micro-actuators with respect to their dimensional footprint is a promising feature that can be further improved. The current results showed positioning accuracy not higher than $1 \%$ of the micro-actuator's total length. The integration of such actuators was proven in a preliminary 2-DoF soft robot, which opens the scope to embed the same in different robotic systems with high dimensional constraints and demanding positioning accuracies.

As an application example, the planar micro-actuator is fixed to a silicone tube of $1 \mathrm{~mm}$ diameter using a heat-shrinkable sleeve at the lower end, as depicted in Figure 10. An optical fiber linked to a laser generator is placed inside the tube. The system is then actuated and the laser is steered to a maximum displacement of $5 \mathrm{~mm}$. This proves motion capabilities of PPy-based micro-actuator notwithstanding its softness. Thus, using a soft actuator while generating sufficient output force provides medical/surgical applications with operational yet safe approach. The range of displacement is satisfactory to operate B-scans with common path optical coherence tomography (OCT) compared to MEMS based probes, ${ }^{15}$ and mosaicking with confocal laser endomicroscopy ${ }^{14}$ if one consider the available diameters (up to $300 \mu \mathrm{m}$ for the $\$ 300$ probe) of the recent development from Mauna Kea Technologies.

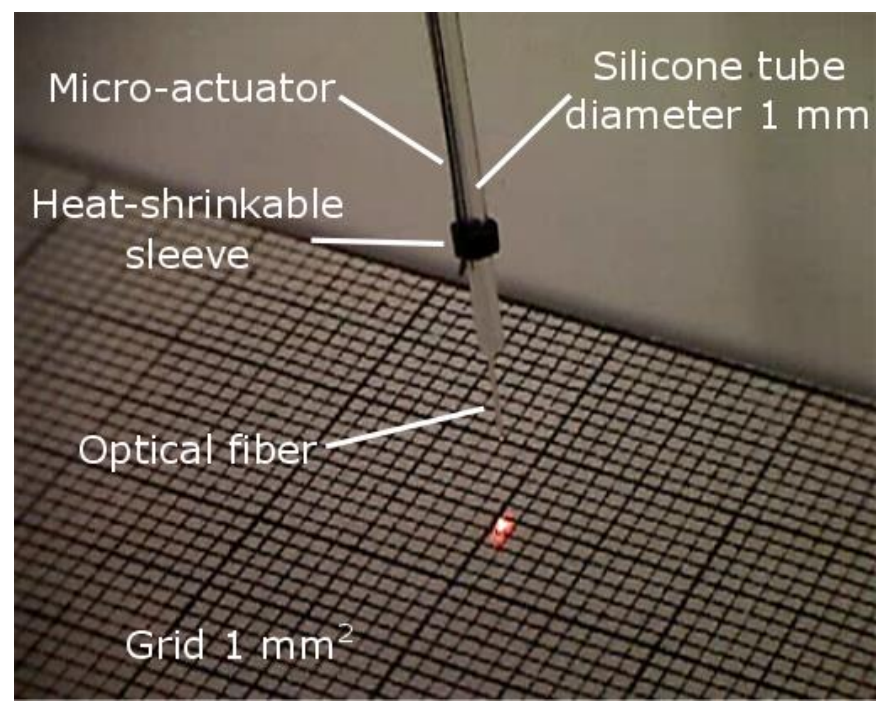

Figure 10: Proof of concept of a PPy-based micro-actuator fixed with a tube embedding optical fiber linked to a laser generator.

Video can be viewed at https://youtu.be/fwIC-UY1s38

In order to increase the integration of PPy micro-actuators on continuum robots, the former could be electrochemically deposited on the tube. This approach is challenging and constitutes an ongoing work in our group. In addition, the use of four electrodes will potentially enable to control the robot link in two DoF. The main technological challenge is to deposit homogenous PPy layers on circular substrates and to separate the required four electrodes.

In conclusion, this paper presents first prototype integrating a PPy-based microactuator in a laser steering scheme. Further, the micro-actuator's maximum force is 
assessed with respect to its polymerization time. Overall, the forces were sufficient to perform $5 \mathrm{~mm}$ displacement with embedded laser and high positioning accuracy.

The movement amplitude can be further increased with longer polymerization time. Conversely, saturation in the thickness growth during the fabrication process could prevent enhancing motion performances and reduce the dynamics. Using Interpenetrating Polymer Networks (IPN) is a promising technique to combine benefits of high-force polymers (e.g. PPy) and high-speed ones (e.g. Polyethylene oxidepolytetrahydrofurane, PEDOT). In order to fulfill multiples objectives (response time, large displacements, high forces), this combination is an open research question, which involves material science/nanotechnology, automatic control, and robotics. Nevertheless, for free-space applications such as laser steering (discussed in this paper), both forces and displacements are satisfactory, while the motion velocity should be improved. In order to do so, one can increase the voltage amplitude limit, at the expense of a lower sustainability of the EAP-based micro-actuators.

The control experiments were led using visual feedback from an external camera. While this configuration was proven effective, its integration in a realistic medical scenario requires further experiments and adaptation. Intraoperative images can be used as feedback, or methods using miniaturized cameras in eye-in-hand configuration can be implemented to provide an endoscopic-like view [KudryavtsevRAL2018]. Further, OCT devices represent suitable candidates such as those from Agiltron (Woburn, Massachusetts, USA) or confocal microscopes from Mauna Kea Technology (Paris, France), both of $0.3 \mathrm{~mm}$ in diameter.

Kudryavtsev AV, Chikhaoui MT, Liadov A, Rougeot P, Spindler F, Rabenorosoa K, Burgner-Kahrs J, Tamadazte B, and Andreff N. Eye-in-Hand Visual Servoing of Concentric Tube Robots. IEEE Robotics and Automation Letters, 2018 (in print).

\section{ACKNOWLEDGMENT}

This work has been supported by the Labex ACTION project (contract "ANR-11-LABX0001-01"), the Equipex ROBOTEX project (contract "ANR-10-EQPX-44-01"), and the French RENATECH network and its FEMTO-ST technological facility.

\section{REFERENCES}

1. Burgner-Kahrs J, Rucker DC, Choset H. Continuum Robots for Medical Applications: A Survey. IEEE Transactions on Robotics, 2015, 31(6), 1261-1280.

2. Carpi F, Smela E. Biomedical Applications of Electroactive Polymer Actuators, Wiley Online Library, 2009.

3. Chikhaoui MT, Rabenorosoa K, Andreff N. Kinematics and Performance Analysis of a Novel Concentric Tube Robotic Structure with Embedded Soft Microactuation. Mechanism and Machine Theory, 2016, 104, 234-254.

4. Chikhaoui MT, Cot A, Rabenorosoa K, Rougeot P, Andreff N. Design and Closed-loop Control of a Tri-layer Polypyrrole based Telescopic Soft Robot. IEEE/RSJ International Conference on Intelligent Robots and Systems, 2016, 1145-1150.

5. Chikhaoui MT, Cot A, Rabenorosoa K, Rougeot P, Andreff N. Towards Biocompatible Conducting Polymer Actuated Tubes for Intracorporeal Laser Steering. Hamlyn Symposium on Medical Robotics, 2017, 79-80. 
6. Cianchetti M, Ranzani T, Gerboni G, De Falco I, Laschi C, Menciassi A. STIFFFLOP surgical manipulator: Mechanical design and experimental characterization of the single module. IEEE/RSJ International Conference on Intelligent Robots and Systems, 2013, 3576-3581.

7. Cot A, Chikhaoui MT, Rougeot P, Rabenorosoa K, Andreff N. Synthesis, Encapsulation, and Performance Analysis of Large Deformation Tri-Layer Polypyrrole Actuator. IEEE International Conference on Advanced Intelligent Mechatronics, 2016, 436-441.

8. Couture T, Szewczyk J. Design and experimental validation of an active catheter for endovascular navigation. Journal of Medical Devices, 2017, 12(1), 011003-011003-12.

9. Gairhe B, Alici G, Spinks GM, Cairney JM. Synthesis and Performance Evaluation of Thin Film PPy-PVDF Multilayer Electroactive Polymer Actuators. Sensors \& Actuators A: Physical, 2011, 165(2), 321-328.

10. Gilbert HB, Rucker DC, Webster III RJ. Concentric Tube Robots: The State of the Art and Future Directions. In: Inaba M., Corke P. (eds) Robotics Research. Springer Tracts in Advanced Robotics, 2016, vol 114.

11. Ha J, Park F, Dupont P. Elastic Stability of Concentric Tube Robots Subject to External Loads. IEEE Transactions on Biomedical Engineering, 2015, 63(6), 11161128.

12. Ikeuchi M, Ikuta K. Development of pressure-driven micro active catheter using membrane micro emboss following excimer laser ablation (MeME-X) process. IEEE International Conference on Robotics and Automation, 2009, 4469-4472.

13. Nam J, Kim Y, Jang G. Resonant Piezoelectric Vibrator with High Displacement at Haptic Frequency for Smart Devices. IEEE/ASME Transactions on Mechatronics, 2016, 21(1), 394-401.

14. Rosa B, Erden MS, Vercauteren T, Herman B, Szewczyk, J, Morel G. Building Large Mosaics of Confocal Endomicroscopic Images using Visual Servoing. IEEE Transactions on Bio-Medical Engineering, 2013, 60(4), 1041-1049.

15. Sun J, Xie H. MEMS-based Endoscopic Optical Coherence Tomography. International Journal of Optics, 2011, Article ID 825629.

16. Webster III RJ, Jones BA. Design and Kinematic Modeling of Constant Curvature Continuum Robots: A Review. The International Journal of Robotics Research, 2010, 29(13), 1661-1683.

17. Xu K, Goldman RE, Ding J, Allen PK, Fowler DL, Simaan N. System Design of an Insertable Robotic Effector Platform for Single Port Access (SPA) Surgery. IEEE/RSJ International Conference on Intelligent Robots and Systems, 2009, 5546-5552.

18. Yamaura M, Hagiwara T, Iwata K. Enhancement of Electrical Conductivity of Polypyrrole Film by Stretching: Counter-ion Effect. Synthetic Metals, 1988, 26(3), 209-224.

19. Yip MC, Camarillo DB. Model-Less Hybrid Position/Force Control: A Minimalist Approach for Continuum Manipulators in Unknown, Constrained Environments. IEEE Robotics and Automation Letters, 2016, 1(2), 844-851. 